\title{
Le coton à El Deir
}

Premières observations sur l'existence d'une nouvelle fibre textile dans l'oasis de Kharga (désert occidental égyptien, $\mathrm{V}^{\mathrm{e}}$ siècle $\mathrm{AEC}-\mathrm{V}^{\mathrm{e}}$ siècle EC)

Cotton at el-Deir. First observations on a new textile fibre in the Kharga oasis (Egyptian Western desert, $5^{\text {th }}$ century BCE-5 $5^{\text {th }}$ century CE)

\section{Fleur Letellier-Willemin}

\section{OpenEdition}

\section{Journals}

Édition électronique

URL : http://journals.openedition.org/ethnoecologie/4283

DOI : $10.4000 /$ ethnoecologie.4283

ISSN : 2267-2419

Éditeur

Laboratoire Eco-anthropologie et Ethnobiologie

Référence électronique

Fleur Letellier-Willemin, «Le coton à El Deir », Revue d'ethnoécologie [En ligne], 15 | 2019, mis en ligne le , consulté le 30 octobre 2019. URL : http://journals.openedition.org/ethnoecologie/4283 ; DOI :

10.4000/ethnoecologie.4283

Ce document a été généré automatiquement le 30 octobre 2019.

\section{c) (1) $९$}

Revue d'ethnoécologie est mis à disposition selon les termes de la licence Creative Commons Attribution - Pas d'Utilisation Commerciale - Pas de Modification 4.0 International. 


\section{Le coton à El Deir}

Premières observations sur l'existence d'une nouvelle fibre textile dans l'oasis de Kharga (désert occidental égyptien, $\mathrm{v}^{\mathrm{e}}$ siècle $\mathrm{AEC}-\mathrm{V}^{\mathrm{e}}$ siècle $\mathrm{EC}$ )

Cotton at el-Deir. First observations on a new textile fibre in the Kharga oasis

(Egyptian Western desert, $5^{\text {th }}$ century BCE-5 $5^{\text {th }}$ century CE)

\section{Fleur Letellier-Willemin}

\section{Introduction}

1 À el-Deir, site du nord-est de l'oasis de Kharga (désert occidental égyptien), le coton occupe une place bien particulière dans l'ensemble du corpus textile mis au jour, principalement composé de fibres de lin et de laine. Attesté dans l'oasis à partir du II siècle EC selon les textes (Tallet et al. 2012 : 126), le coton fut découvert sous différentes formes, chacune attestant une étape particulière de la chaîne opératoire textile (Bouchaud et al. ce volume). Cet article a pour objectif de rapporter la présence du coton sur le site, en se basant sur les données de terrain mises en lumière par les fouilles de la mission française d'el-Deir, dirigée par Gaëlle Tallet.

2 Les divers travaux menés sur le vaste site d'el-Deir ont révélé des restes de coton dans tous les ensembles archéologiques: dans les nécropoles dites «traditionnelles », datées $\mathrm{du} \mathrm{VI}^{\mathrm{e}}$ siècle $\mathrm{AEC}$ à la fin du $\mathrm{III}^{\mathrm{e}}$ siècle EC environ, dans la nécropole dite "chrétienne " de la fin $\mathrm{du} \mathrm{III}^{\mathrm{e}}$ au VI $\mathrm{I}^{\mathrm{e}}$ siècle EC, dans l'atelier d'embaumeur, ainsi que dans l'enceinte du temple et sur son pourtour ${ }^{1}$. Seuls les sondages effectués à l'emplacement de la forteresse ne comportaient aucun reste de coton.

Prises dans leur ensemble, ces données permettent d'illustrer un aspect important de la consommation textile dans l'oasis de Kharga, et permettent d'émettre des hypothèses sur l'évolution des pratiques agricoles et artisanales qui, en Égypte, marque la période charnière de l'Antiquité tardive. 
Découvertes de terrains : graines, capsules, fils, pelotes, textiles et cordelettes de coton

Graines et capsules (Figures $1 a$ et $1 b^{2}$ )

Figure 1a : Graines de coton, couche 4227 du temple

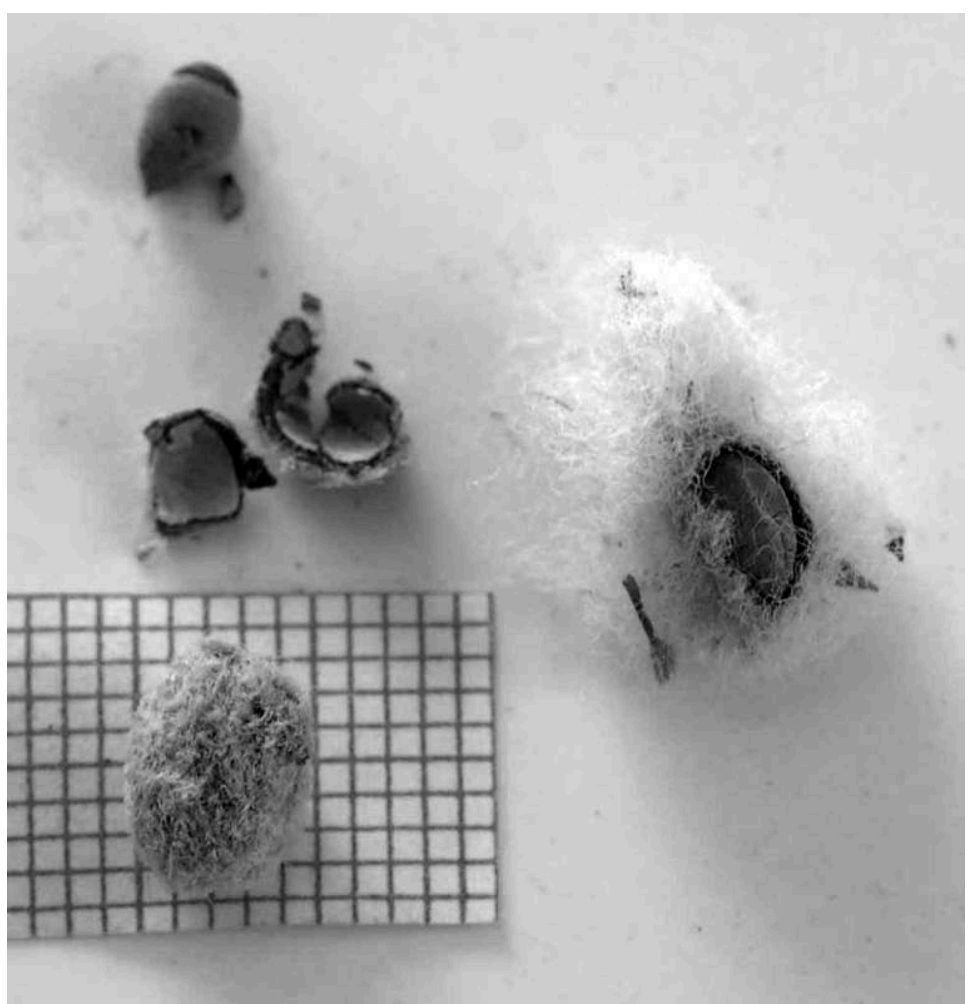

Photo F. Letellier-Willemin / Mission archéologique d'el-Deir

Figure 1b: Capsules de la tombe N1

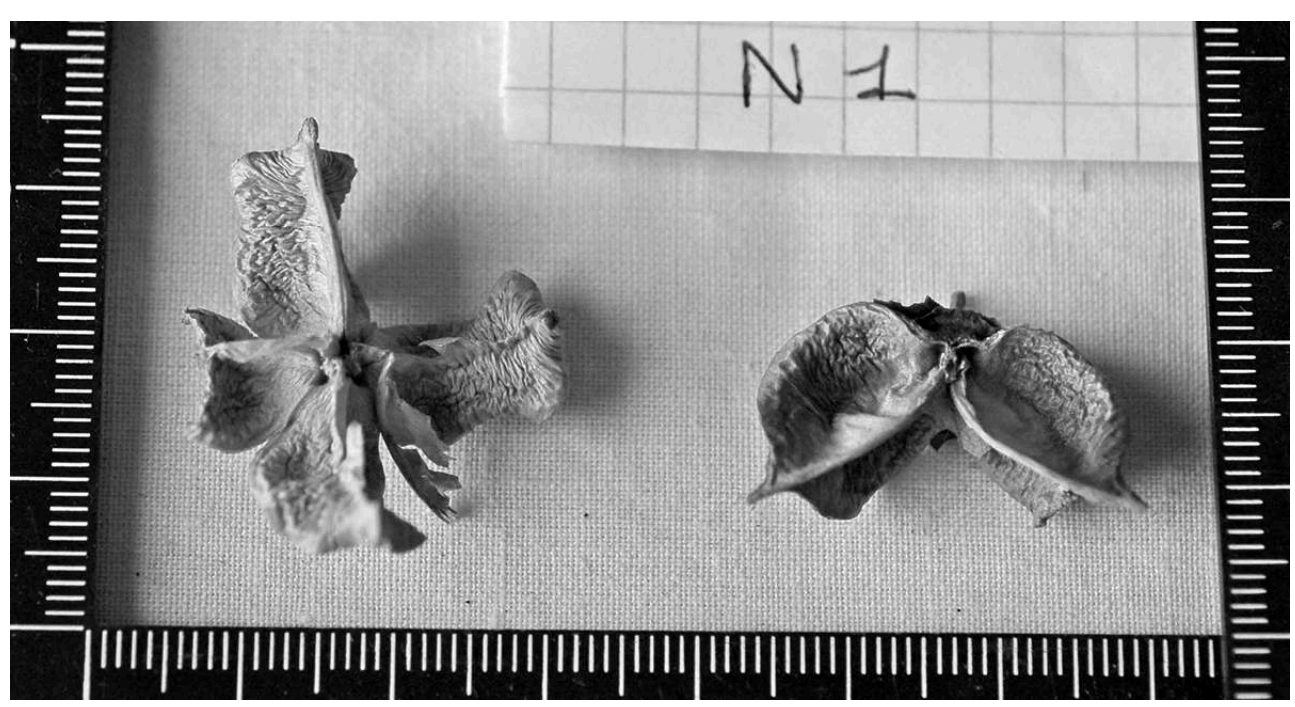

Photo F. Letellier-Willemin / Mission archéologique d'el-Deir 
Figure 2 : Détail des découvertes de capsules et graines de coton à El Deir

\begin{tabular}{c|c|c|c|}
\hline \multicolumn{1}{c|}{ Site } & Type & Contexte & Date \\
\cline { 2 - 4 } $\begin{array}{c}\text { Nécropole } \\
\text { Nord }\end{array}$ & 2 capsules de coton & $\begin{array}{c}\text { Tombe N.1. } \\
\text { Parmi des textiles en vrac. }\end{array}$ & $\begin{array}{c}\text { Epoque gréco-romaine } \\
\text { (basée sur d'autres tombes moins perturbées du cimetière) }\end{array}$ \\
\cline { 2 - 4 } Temple & $\begin{array}{c}\text { Graines associées à } \\
\text { quelques fils de coton }\end{array}$ & $\begin{array}{c}\text { Pièce X, } \\
\text { couche 4227. }\end{array}$ & Epoque gréco-romaine \\
\hline
\end{tabular}

4 L'association du végétal (graines) et des fils au sein d'un même contexte devant le temple (Figure 2) permet d'envisager la culture locale du coton, ou en tout cas le traitement sur place de la fibre brute. Nous pensons en particulier au travail de nettoyage et de préparation du coton (égrainage et cardage éventuel) et au filage. L'organisation des premières étapes de la chaine opératoire cotonnière (Bouchaud et al. ce volume) constitue un point essentiel à préciser.

\section{Fils et pelotes (Figure 3)}

Figure 3 : Pelote de coton

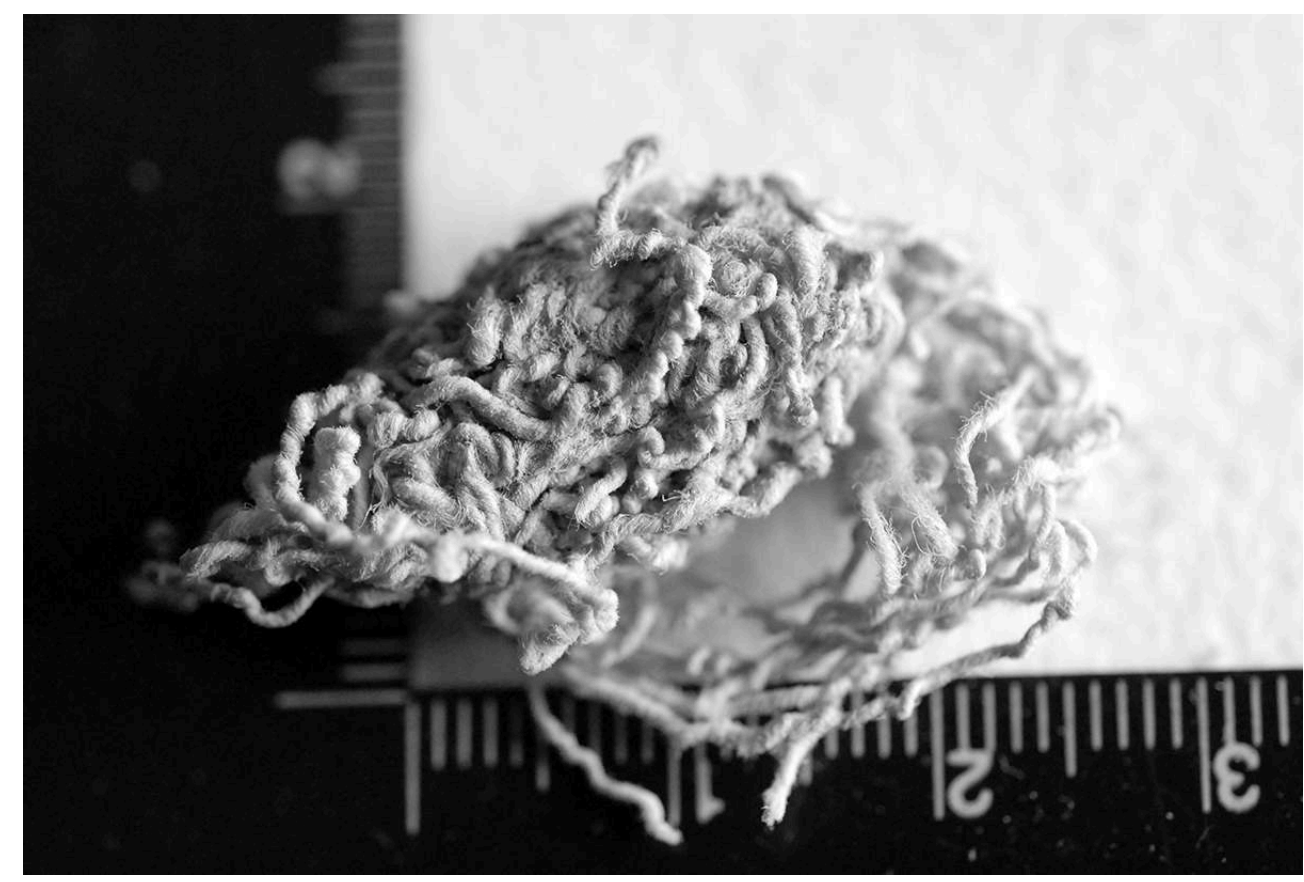

Photo F. Letellier-Willemin / Mission archéologique d'el-Deir

5 Tous les fils étudiés sont de torsion $-\mathrm{S}$, c'est-à-dire filés dans le sens contraire des aiguilles d'une montre. L'angle de torsion est toujours très élevé, se situant entre $60^{\circ}$ et $90^{\circ}$. En raison de cette haute torsion, difficilement gérable, les fils présentent un aspect très hétérogène, avec un diamètre variable et irrégulier. Difficiles à tisser, leurs défauts se traduisent dans le rendu des textiles, dont la structure en armure toile est pour la plupart d'entre eux très irrégulière. Ce premier constat technique, visible dès le début de l'analyse textile, nous amène à questionner la nature des outils utilisés et l'expérience des fileurs.

6 Des fils $\mathrm{S}_{2} \mathrm{Z}^{3}$ sont également mis en évidence, employés pour maintenir en place ourlets, coutures, rapiéçages, et reprises, ainsi que sur des textiles divers en coton, en 
lin ou en laine. L'utilisation de tels fils retors est très fréquemment relevée, aussi bien sur les tissus en laine qu'en lin, et spécialement courante dans le cas de fils de lin employés en couture (Figure 4).

Figure 4 : Fil Z2S de couture

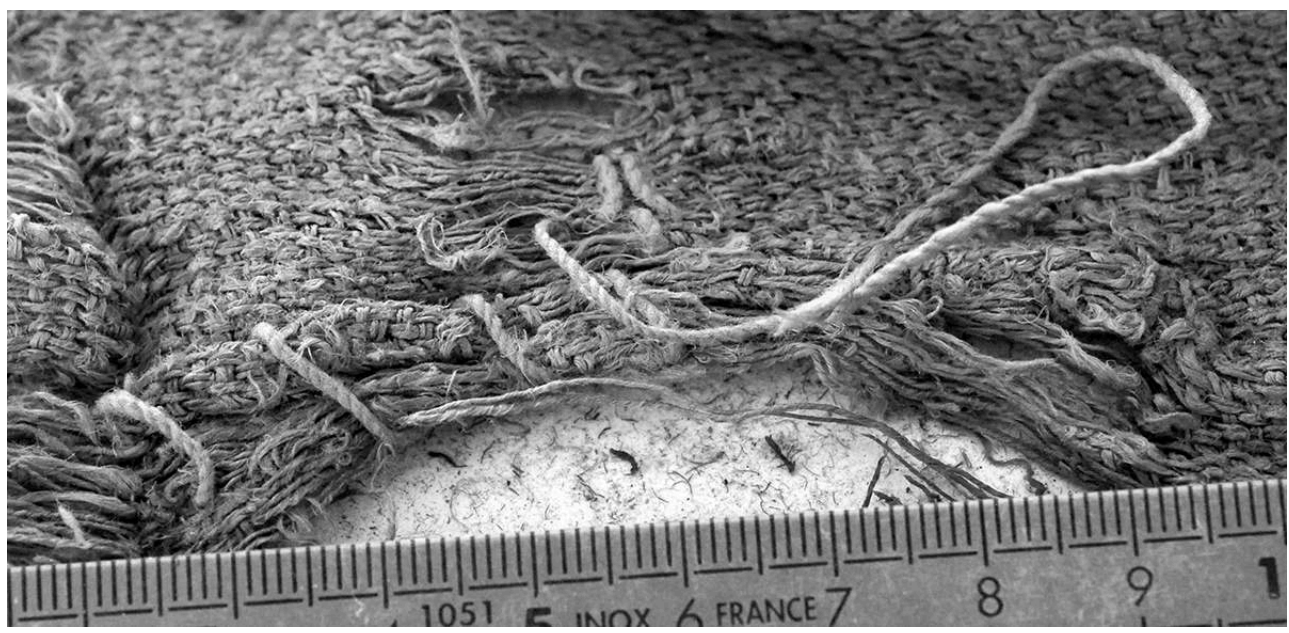

Photo F. Letellier-Willemin / Mission archéologique d'el-Deir

7 Ces fragments de fils de coton furent trouvés dans toutes les nécropoles d'el-Deir, où ils viennent compléter la découverte de quelques rares pelotes. La plus conséquente de ces pelotes provient de la nécropole dite « chrétienne », découverte dans un tas de textiles en vrac. Ces objets, bien que rares, reflètent les activités textiles qui émaillaient la vie quotidienne des habitants du site.

\section{Textiles}

Sur l'ensemble des découvertes textiles réalisées au cours des 18 années de fouilles à elDeir, il n'a pas été trouvé de tissu de coton complet. Réduits à l'état de fragments, les tissus en coton proviennent majoritairement des nécropoles et ne représentent qu'une part très minoritaire du corpus textile, majoritairement composé de lin. Leur analyse détaillée permet d'affirmer qu'il ne s'agit pas de textiles neufs, utilisés en linceuls comme certains textiles en lin des nécropoles, mais de pièces maintes fois réutilisées lors de la vie quotidienne et trouvant leur dernier usage dans la tombe.

9 Leur aspect varie selon leurs qualités techniques. Ces différents types de production dépendent en grande partie de la maitrise du filage et du tissage, et nous interroge à nouveau sur la nature des outils et le degré d'expérience des tisserands (Yvanez 2016). Les quelques exemples suivants illustrent la diversité de ce groupe textile :

- Dans la nécropole Nord : un fil de coton est utilisé dans l'ourlet et la couture d'un même textile vraisemblablement d'usage domestique (Figure 5) ; un simple fil de coton épais constitue le décor de trame d'un fragment (Figure 6); un fragment en lin est rapiécé à l'aide d'un fragment en coton (Figure 7). 
Figure 5 : Ourlet et couture en fils de coton sur textile en coton

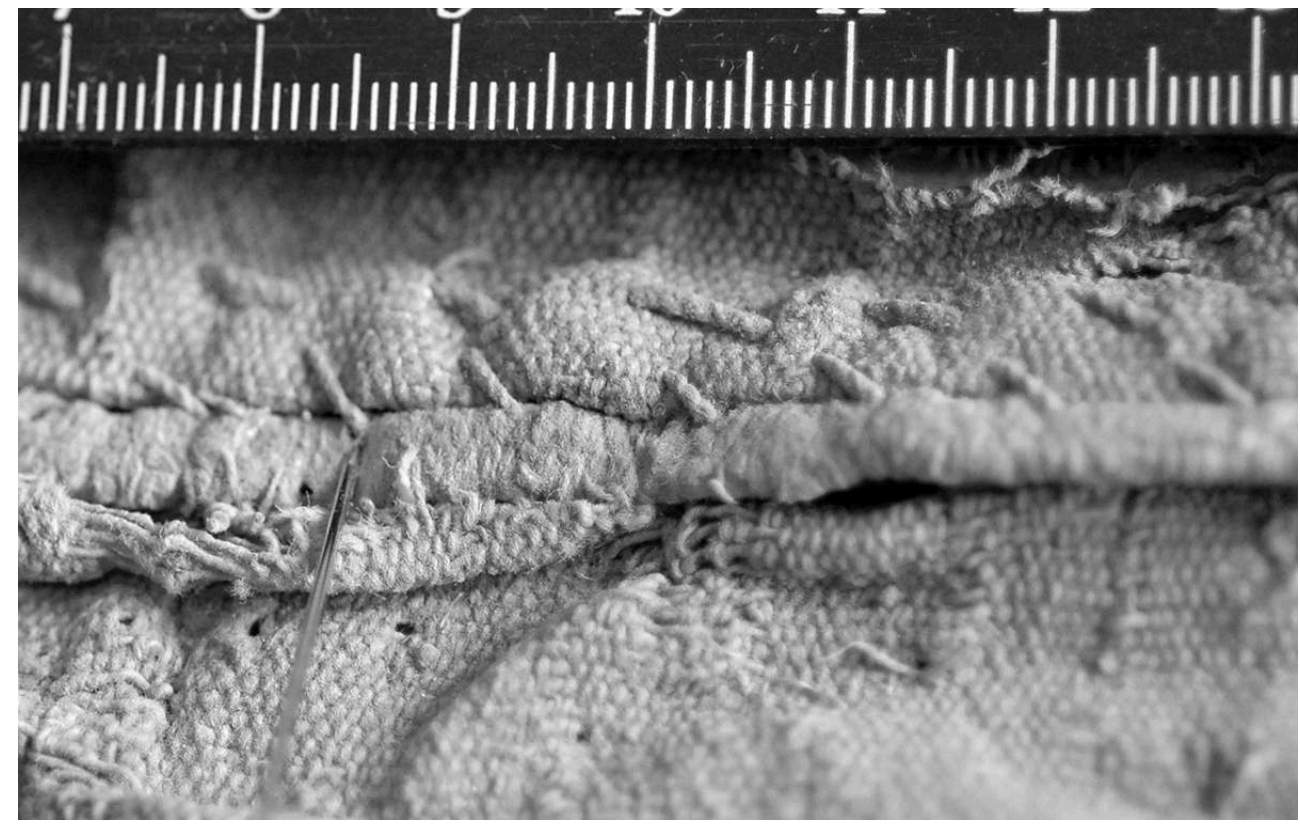

Photo F. Letellier-Willemin / Mission archéologique d'el-Deir

Figure 6 : Décor de trame en coton sur un textile mixte (chaînes en lin et trames en coton)

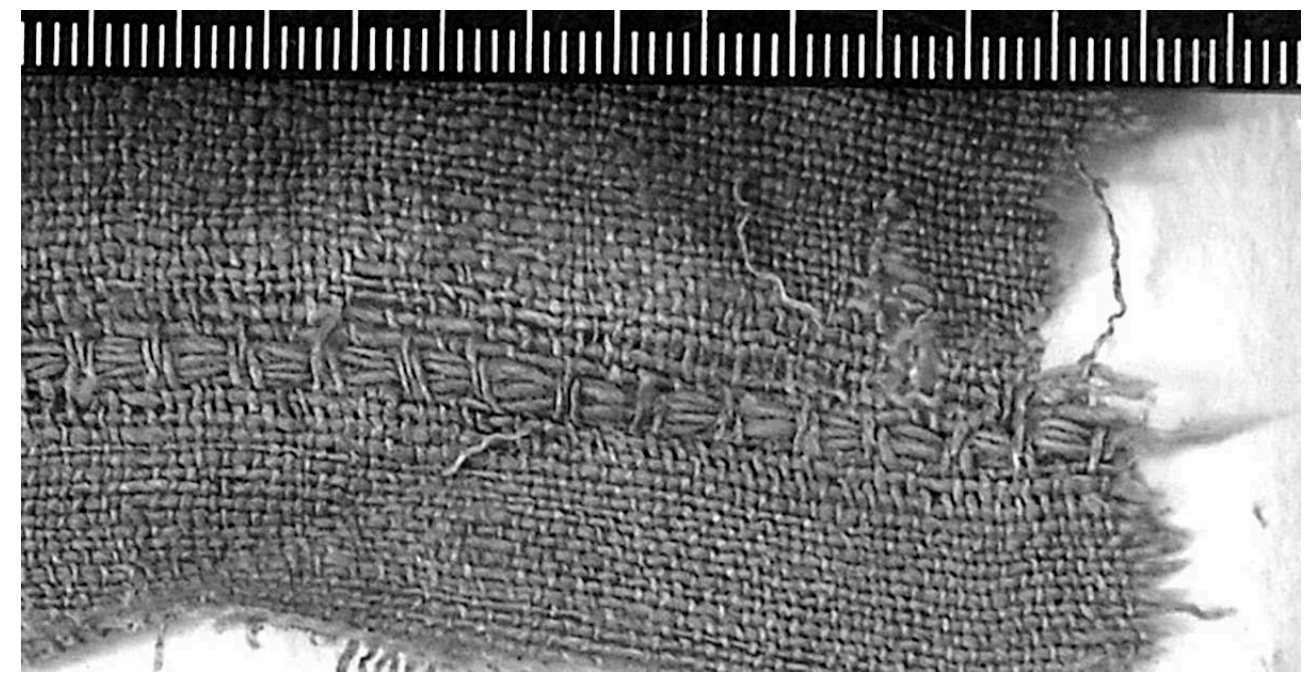

Photo F. Letellier-Willemin / Mission archéologique d'el-Deir 
Figure 7 : Pièce de coton sur du lin

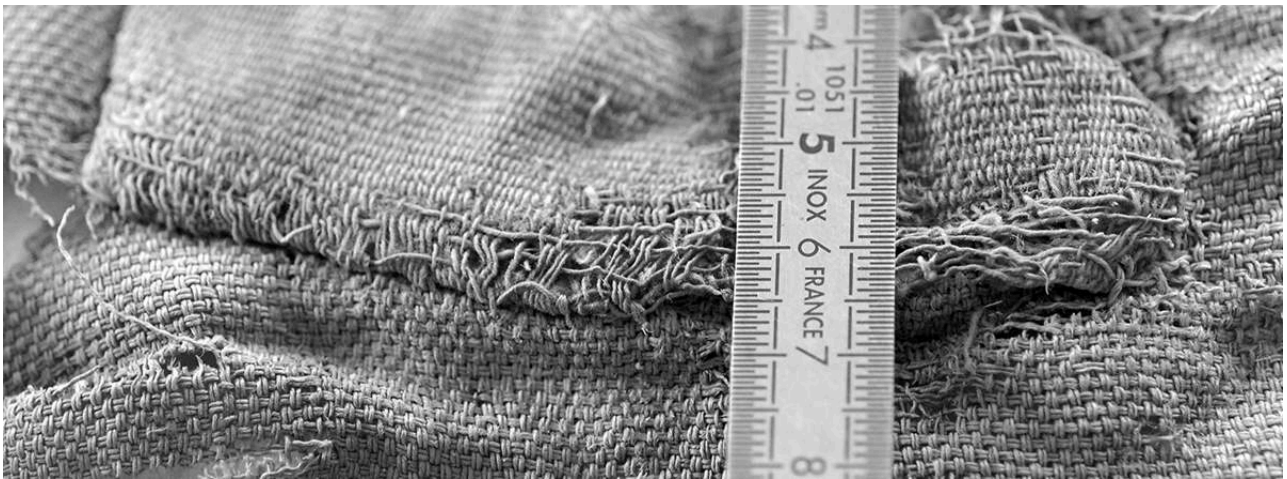

Photo F. Letellier-Willemin / Mission archéologique d'el-Deir

10 - Dans la nécropole Sud et l'atelier d'embaumeur : un fragment en coton couvrait à l'origine l'orifice d'un pot (Figure 8), et un fragment de bande (déchirée dans un textile de bonne qualité, tissé régulièrement) fut vraisemblablement utilisé comme lien (Figure 9).

\section{Figure 8 : Couvercle de pot}

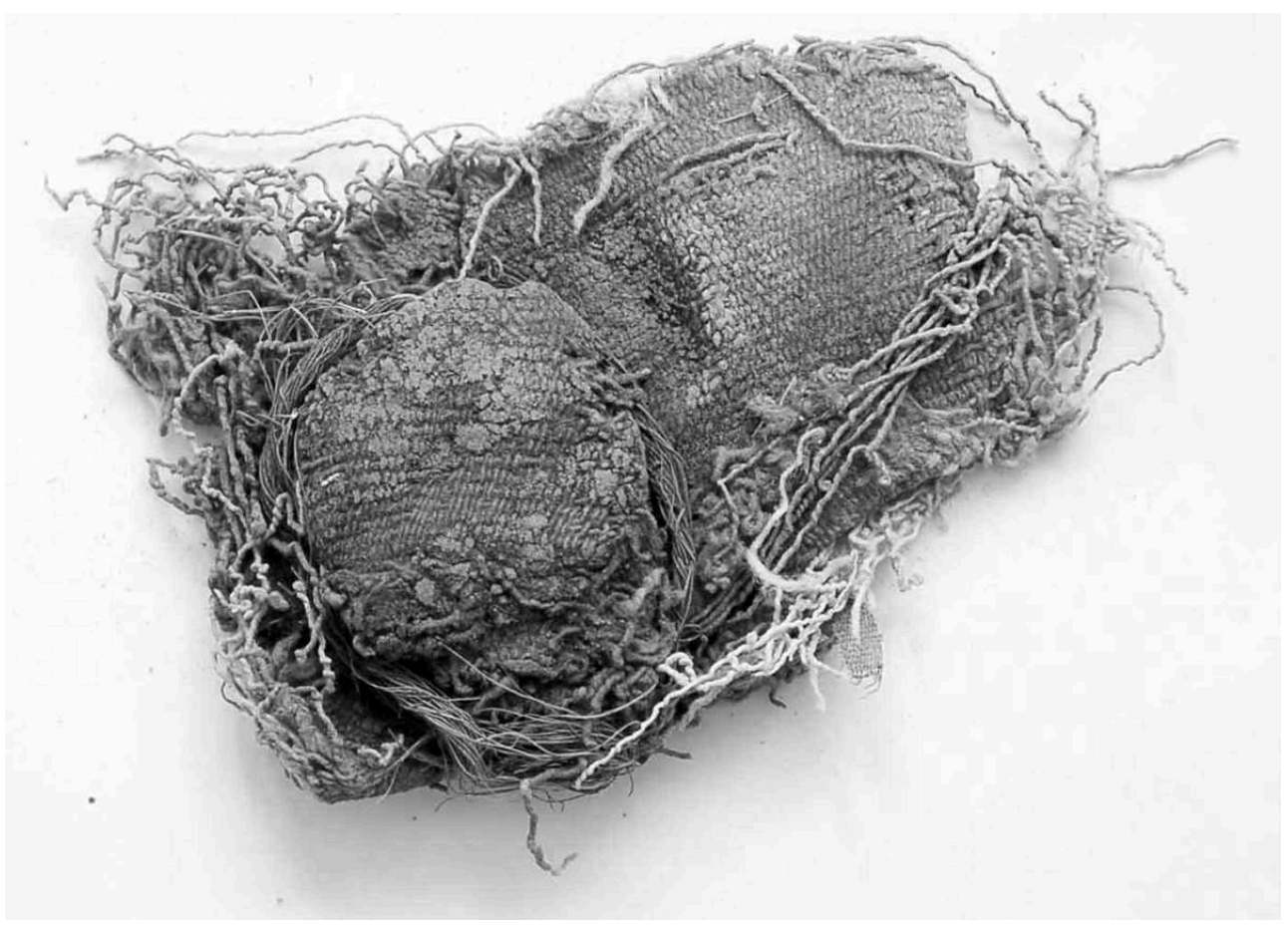

Photo F. Letellier-Willemin / Mission archéologique d'el-Deir 
Figure 9 : Fragment de bande

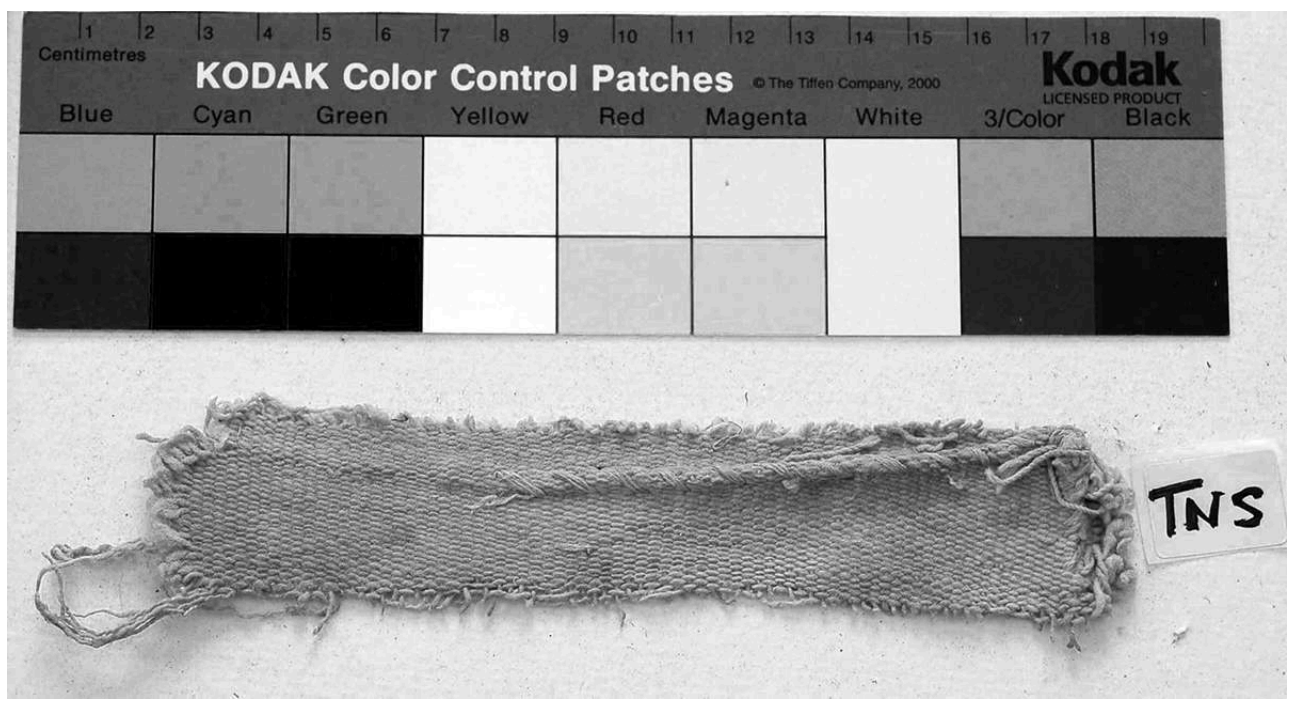

Photo F. Letellier-Willemin / Mission archéologique d'el-Deir

11 - Dans la nécropole Nord-Est : un très petit fragment illustre parfaitement le tissage du coton sur le site (Figure 10). Le coton y est toujours tissé comme la laine, quelle que soit la qualité du tissage. Sur cet exemple, la lisière est celle caractéristique d'un textile en laine, avec des fils et un tissage de qualité.

Figure 10 : Lisière

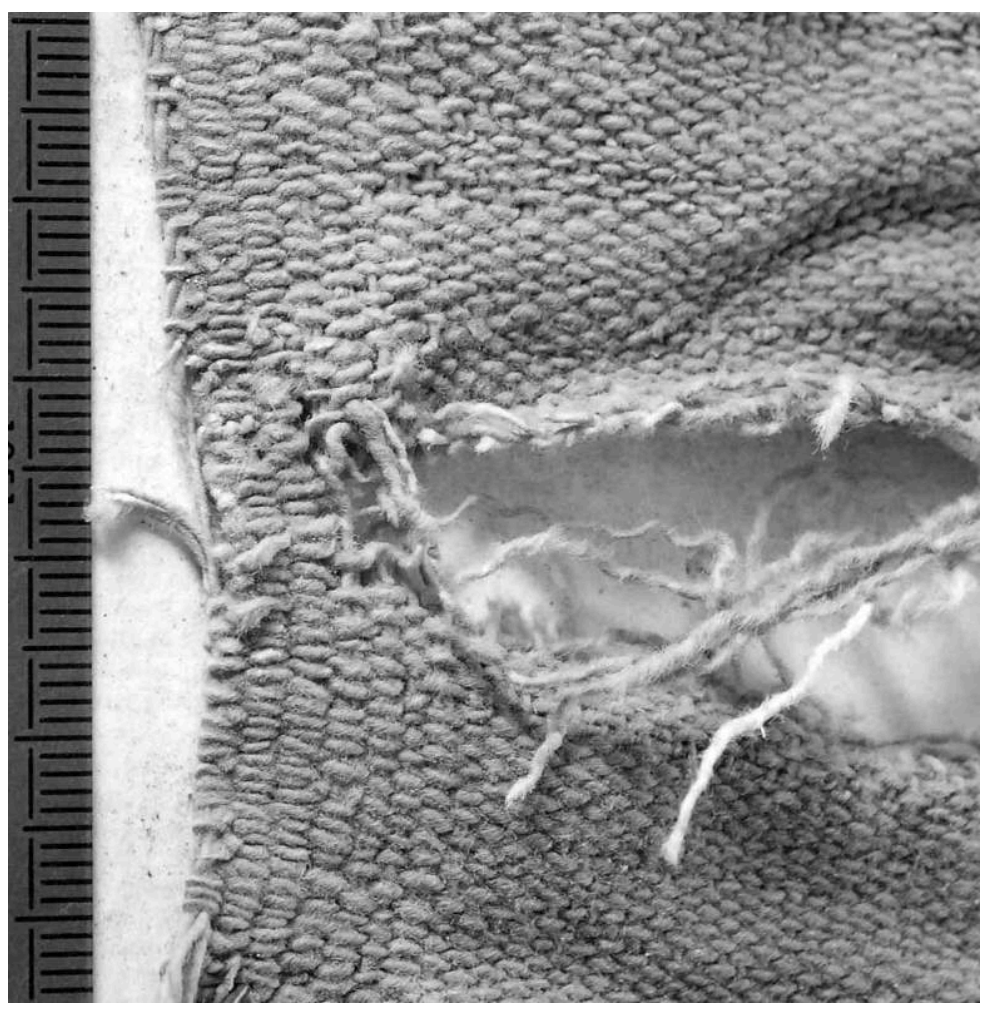

Photo F. Letellier-Willemin / Mission archéologique d'el-Deir

12 - Dans la nécropole Est : ce cimetière a livré un des plus grands fragments en coton du site (Figure 11) ; il est usé, rapiécé et évoque un textile du quotidien, soit vestimentaire, 
soit domestique. Toujours dans la nécropole Est, l'étude des nombreux cartonnages menée par Gaëlle Tallet (Tallet 2015) - a permis de mettre en évidence des supports textiles en coton, parfois en plusieurs épaisseurs, dont certains de bonne qualité (Figure 12).

Figure 11 : Grand fragment usagé

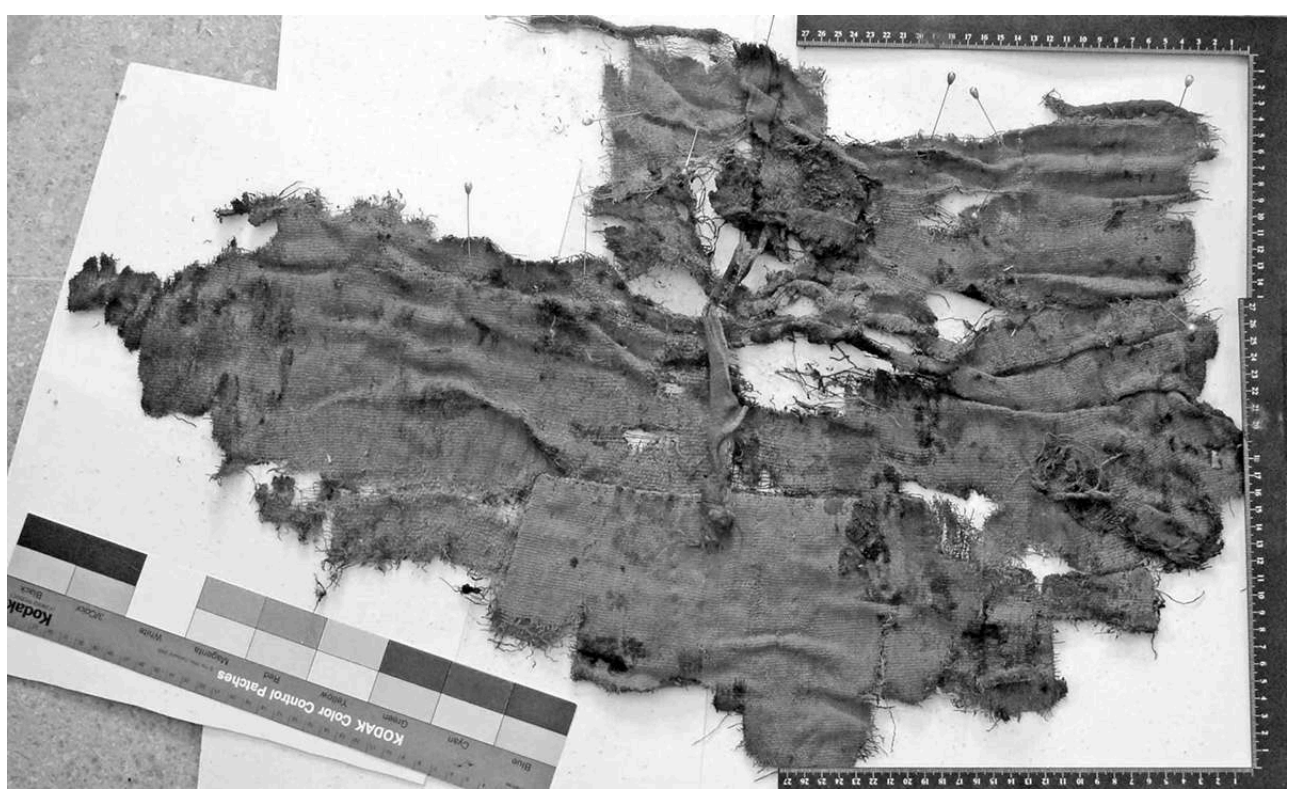

Photo F. Letellier-Willemin / Mission archéologique d'el-Deir

Figure 12 : Supports de cartonnage

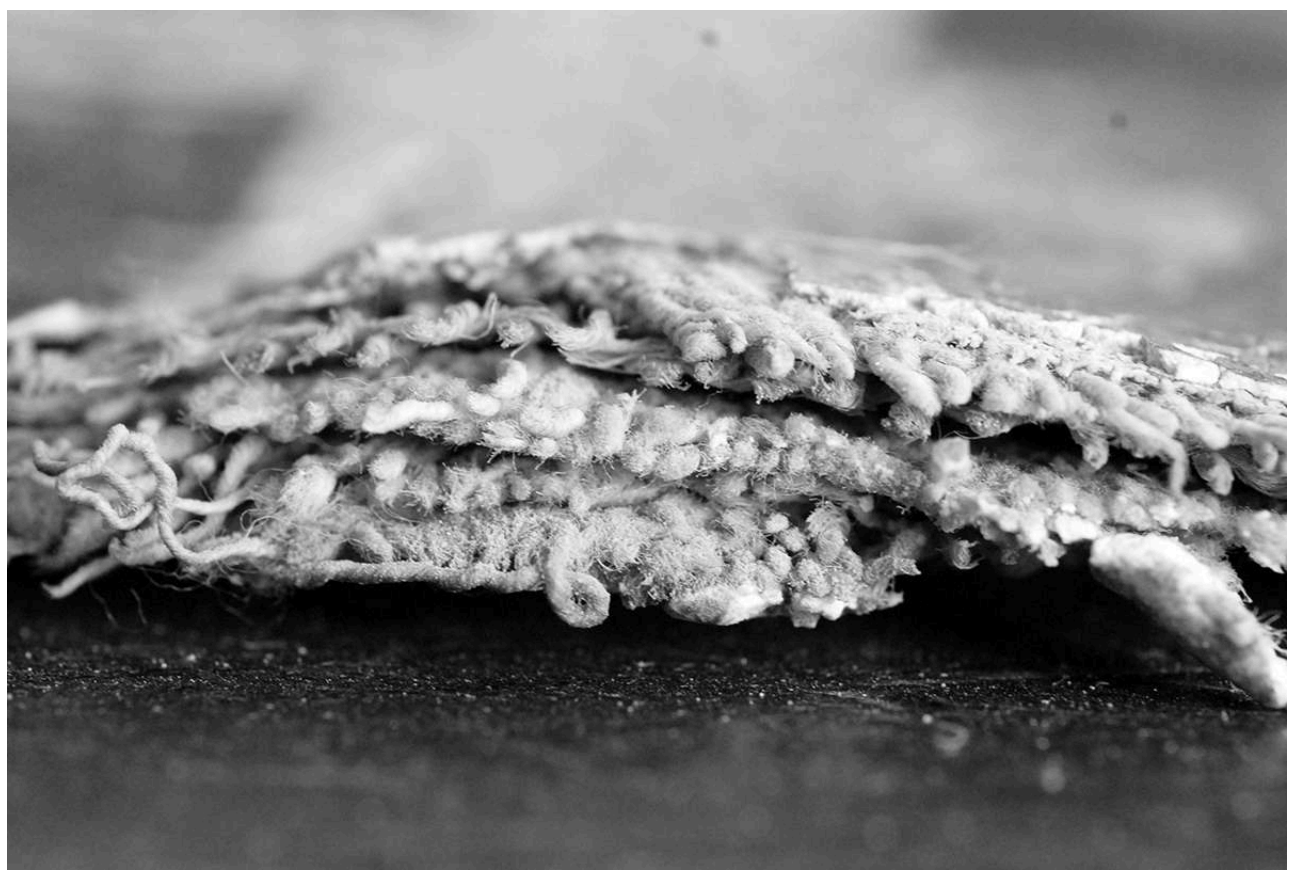

Photo F. Letellier-Willemin / Mission archéologique d'el-Deir

13 - Dans la nécropole dite du «Piton aux Chiens»: les textiles en coton y sont plus nombreux que dans les autres nécropoles, en particulier dans la tombe P5, à 
l'architecture unique sur le site (Dunand 2015 : 152-157). L'un des textiles présente des caractéristiques non connues à ce jour (Figure 13), comparativement aux autres textiles étudiés provenant de Nubie (Yvanez, comm. pers.) et d'Égypte (Wild 2008 : 3-6, Wild 2006: 175-184). Il témoigne d'une grande maîtrise du coton (Letellier-Willemin \& Médard 2012 : 62-71). Des textiles mixtes, coton et lin, y furent également mis au jour. Quelques rares fragments de tissage avec poils (Figure 14) témoignent d'une autre technique, observée sur d'autres sites égyptiens dans le même matériau, comme à Berenike. Cette technique lie de façon intéressante la production textile oasienne à l'artisanat pratiqué dans la vallée du Nil et sur la côte de la Mer Rouge, nous apportant un nouvel éclairage (Wild 2013 : 74-85). Enfin, notons que la tombe P5 contenait de très nombreuses momies de chien, complètes ou fragmentaires, dont aucune ne possédait de textiles en coton.

Figure 13 : Textile unique du Piton aux Chiens

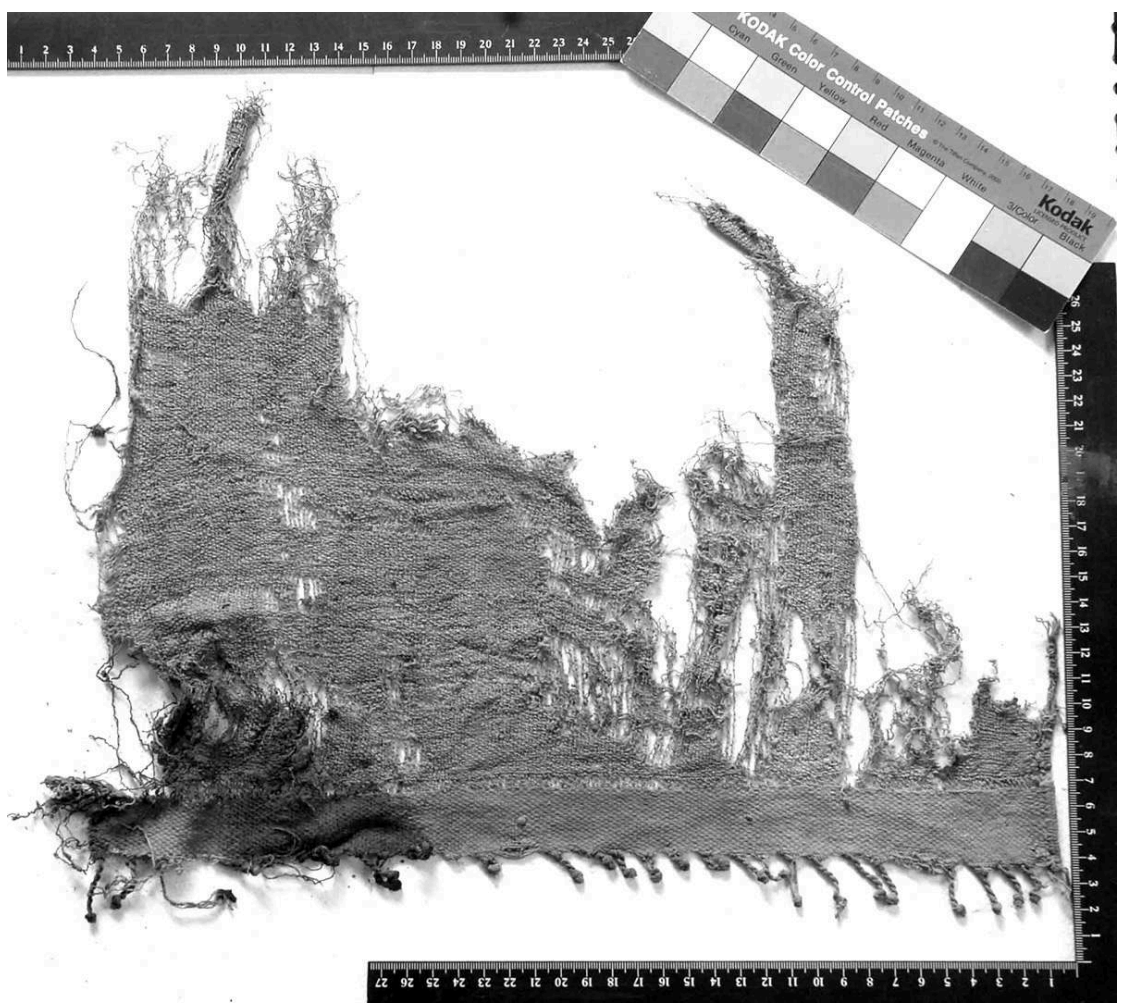

Photo F. Letellier-Willemin / Mission archéologique d'el-Deir 


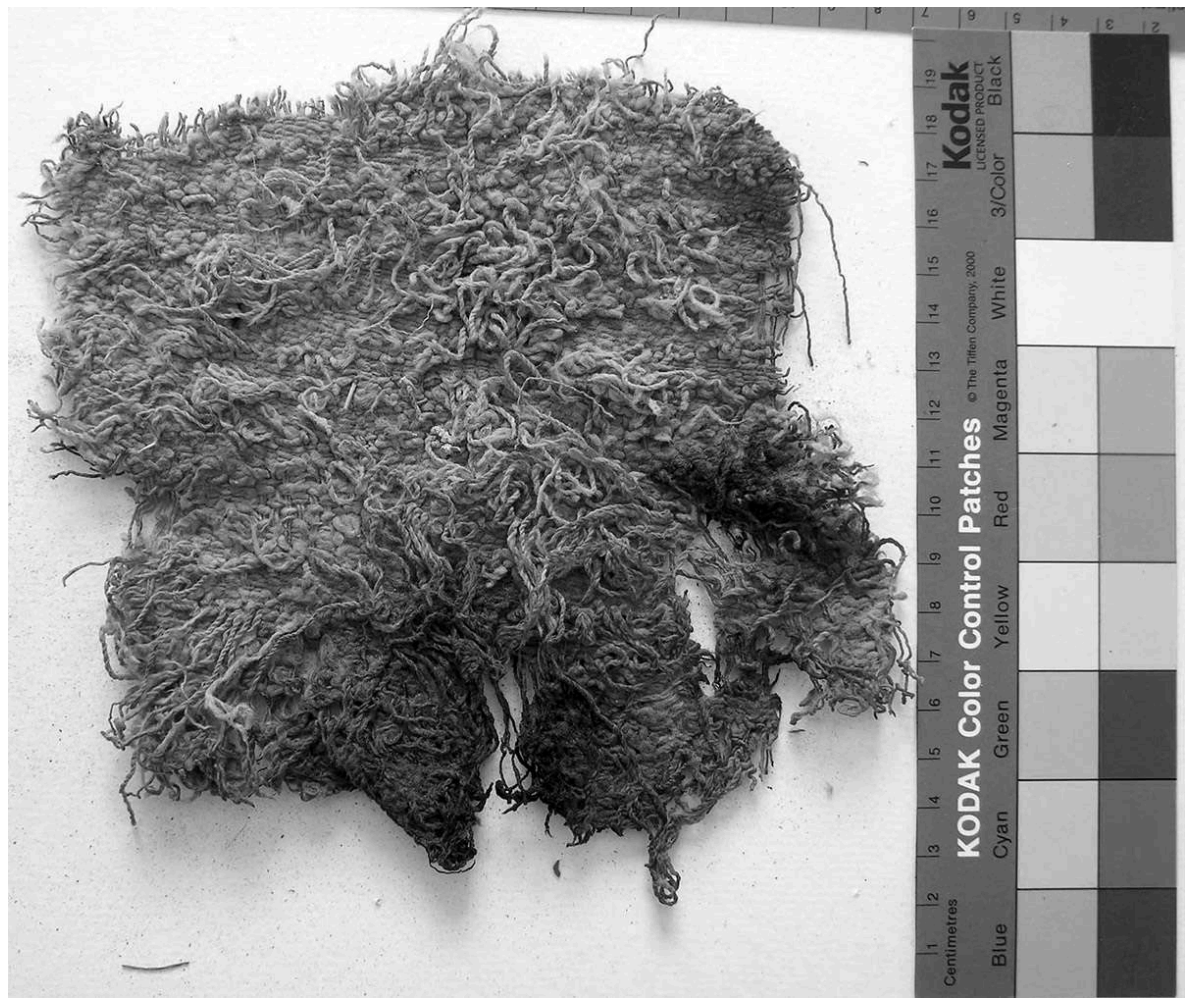

Photo F. Letellier-Willemin / Mission archéologique d'el-Deir

14 - Dans la nécropole Ouest : la fréquence du coton y augmente très nettement. Quelques rares grands textiles incomplets traduisent la réutilisation de textiles du quotidien en linceuls (Figure 15). À nouveau nous mettons en évidence différentes qualités textiles : des textiles très épais à usage domestique, et des textiles de qualité à l'usage plus vraisemblablement vestimentaire, comme des tuniques. Nous constatons fréquemment une bonne qualité textile, et une fibre semblable à celle trouvée dans les autres nécropoles. Certains textiles de coton sont décorés de laine de couleur. Il semble exister une nouvelle qualité de coton, rare : celle présentant un aspect « satiné » (Figure 16). 
Figure 15 : Linceul chrétien

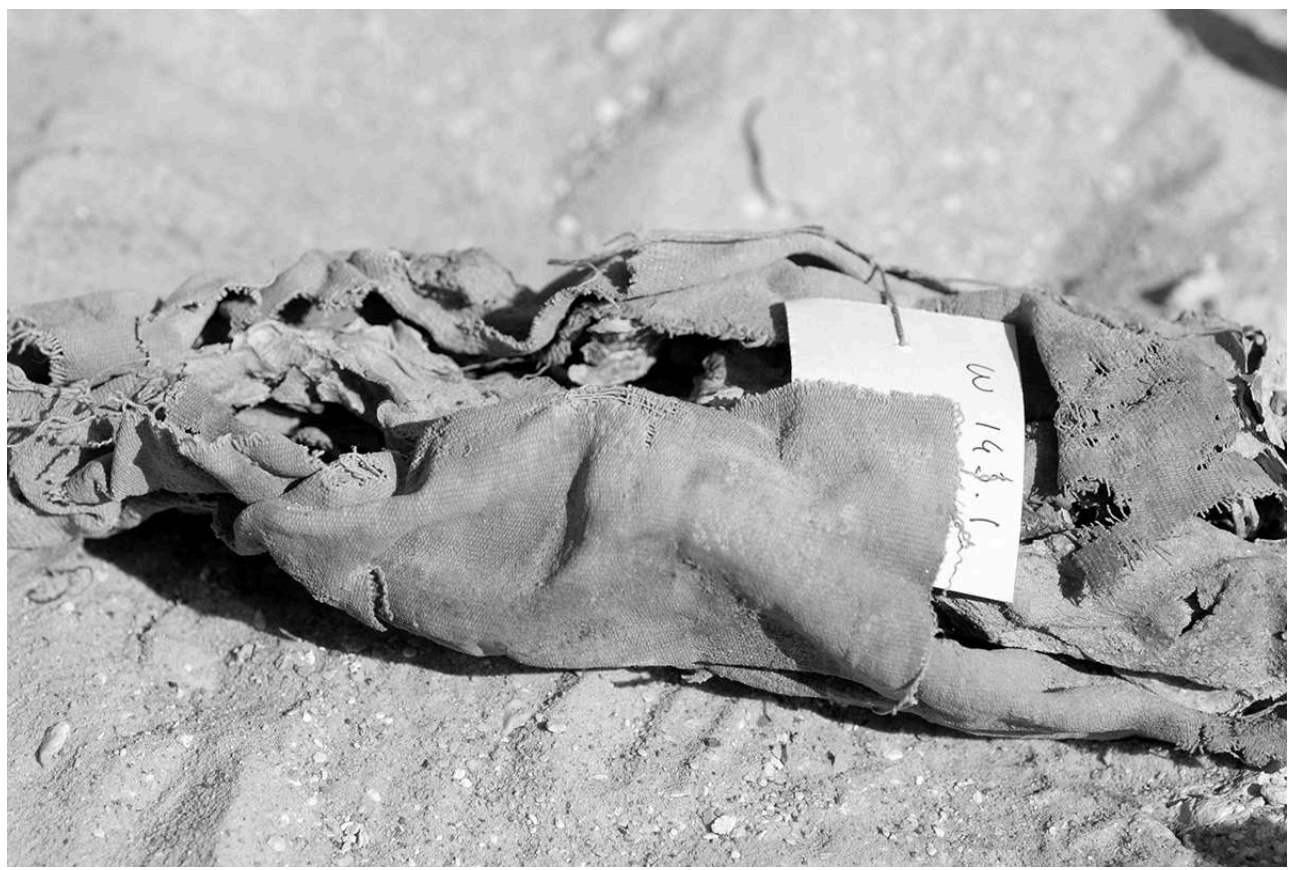

Photo F. Letellier-Willemin / Mission archéologique d'el-Deir

Figure 16 : Aspect « satiné »

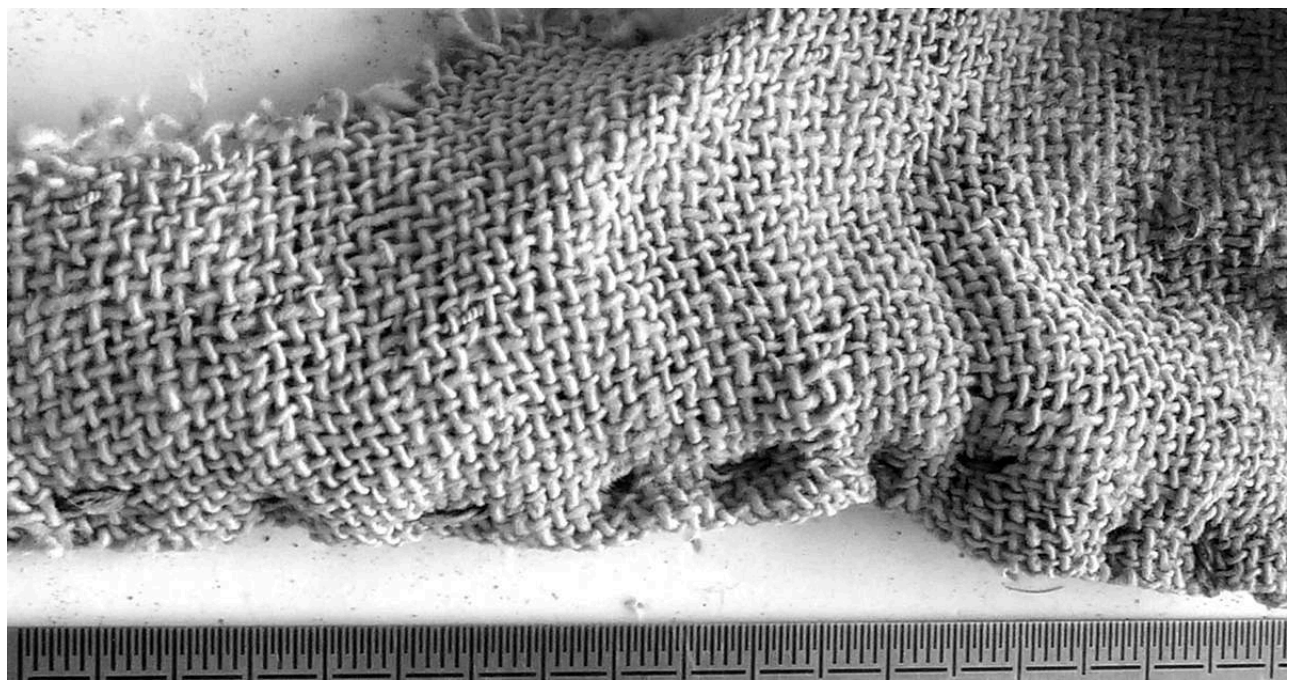

Photo F. Letellier-Willemin / Mission archéologique d'el-Deir

15 - Dans le temple et autour du temple : quelques petits fragments ont été mis en évidence, dans des couches très remaniées.

\section{Cordelettes}

Les cordelettes de coton présentent une grande diversité de fabrication (LetellierWillemin \& Moulherat 2006 : 20-27). Il s'agit toujours de fils de torsion -S, mais ils peuvent être assemblés différemment, et en quantité variable (Figure 17). Des cordelettes ont seulement été mises en évidence par deux fois dans une nécropole 
traditionnelle, la nécropole Nord-Est. Il s'agit d'une part d'un petit tas de cordelettes regroupées, vraisemblablement d'une même provenance, et d'autre part d'une bandelette en lin nouée à une cordelette de coton (Figure 18). Cette découverte surprend car le coton est très rare dans cette nécropole. La grande majorité des cordelettes provient de la nécropole Ouest. Ici, elles remplacent les bandelettes étroites à deux lisières fabriquées en lin, également présentes sur le site et caractéristiques des nécropoles chrétiennes (Heurtel 2003-4), ainsi que les lanières tressées en fibre de palmier.

\section{Figure 17: Cordelettes}

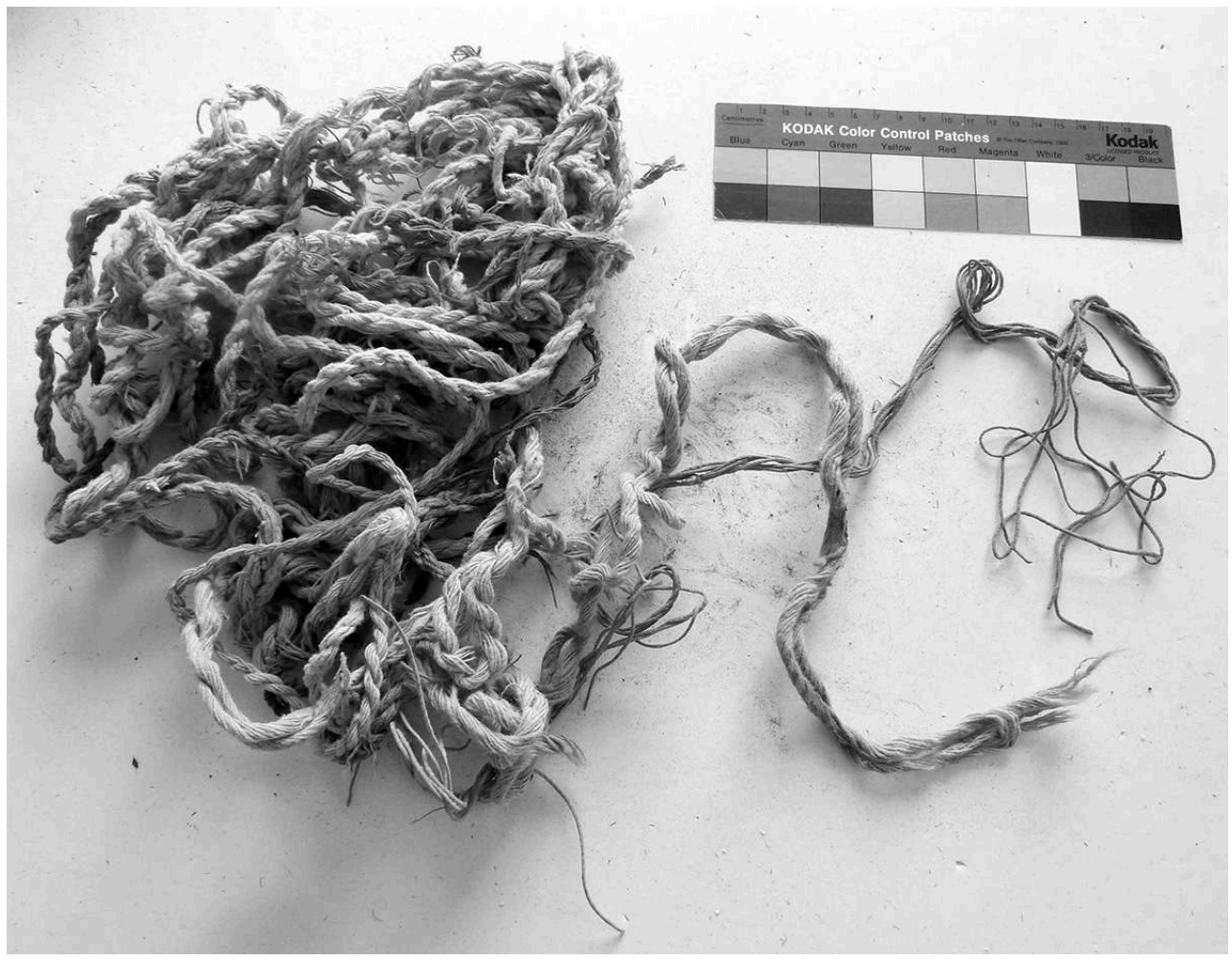

Photo F. Letellier-Willemin / Mission archéologique d'el-Deir 
Figure 18 : Bandelette nouée à une cordelette

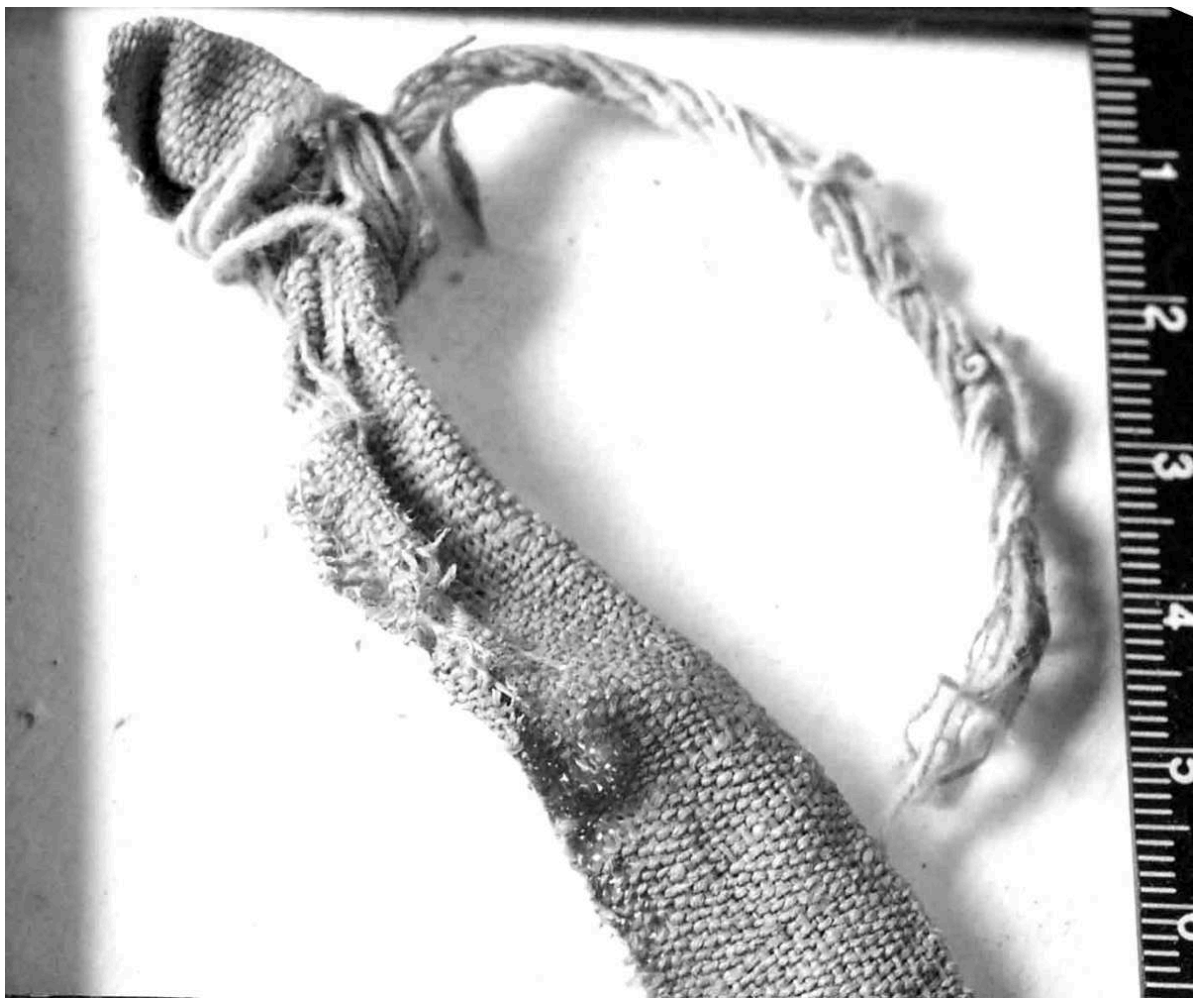

Photo F. Letellier-Willemin / Mission archéologique d'el-Deir

17 Pour une seule momie, la longueur des cordelettes utilisées peut s'avérer très importante: doublées et même triplées autour d'un même corps, les cordelettes peuvent atteindre plusieurs dizaines de mètres de long (Figure 19). Ce constat nous interroge sur leur mode de fabrication et la quantité de matière première et de temps nécessaire à leur manufacture. 
Figure 19 : Momie chrétienne et ses cordelettes

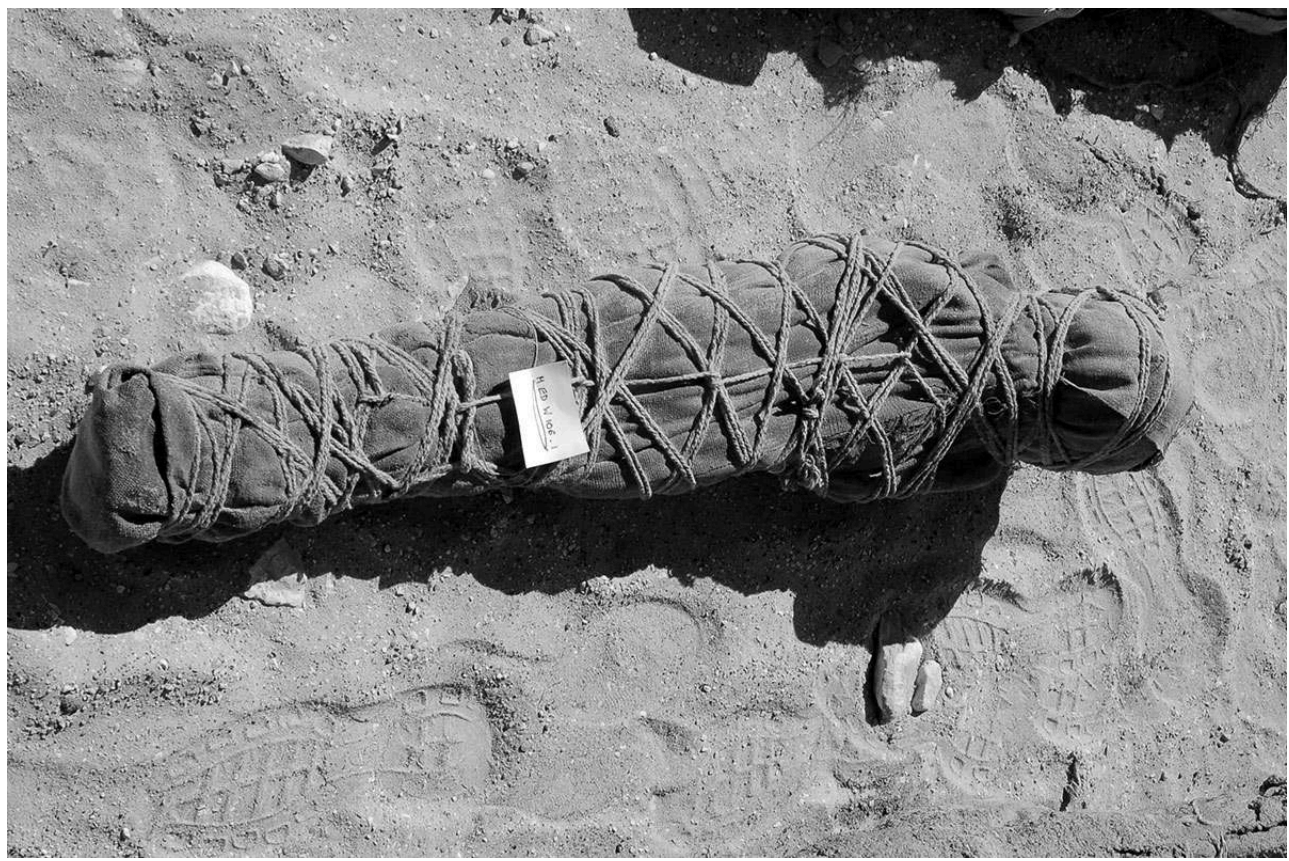

Photo F. Letellier-Willemin / Mission archéologique d'el-Deir

Les cordelettes de coton servent également de support pour la fabrication de bijoux, comme un collier ou un bracelet d'enfant provenant de la tombe W94 (Figure 20).

Figure 20 : Bracelet d'enfant

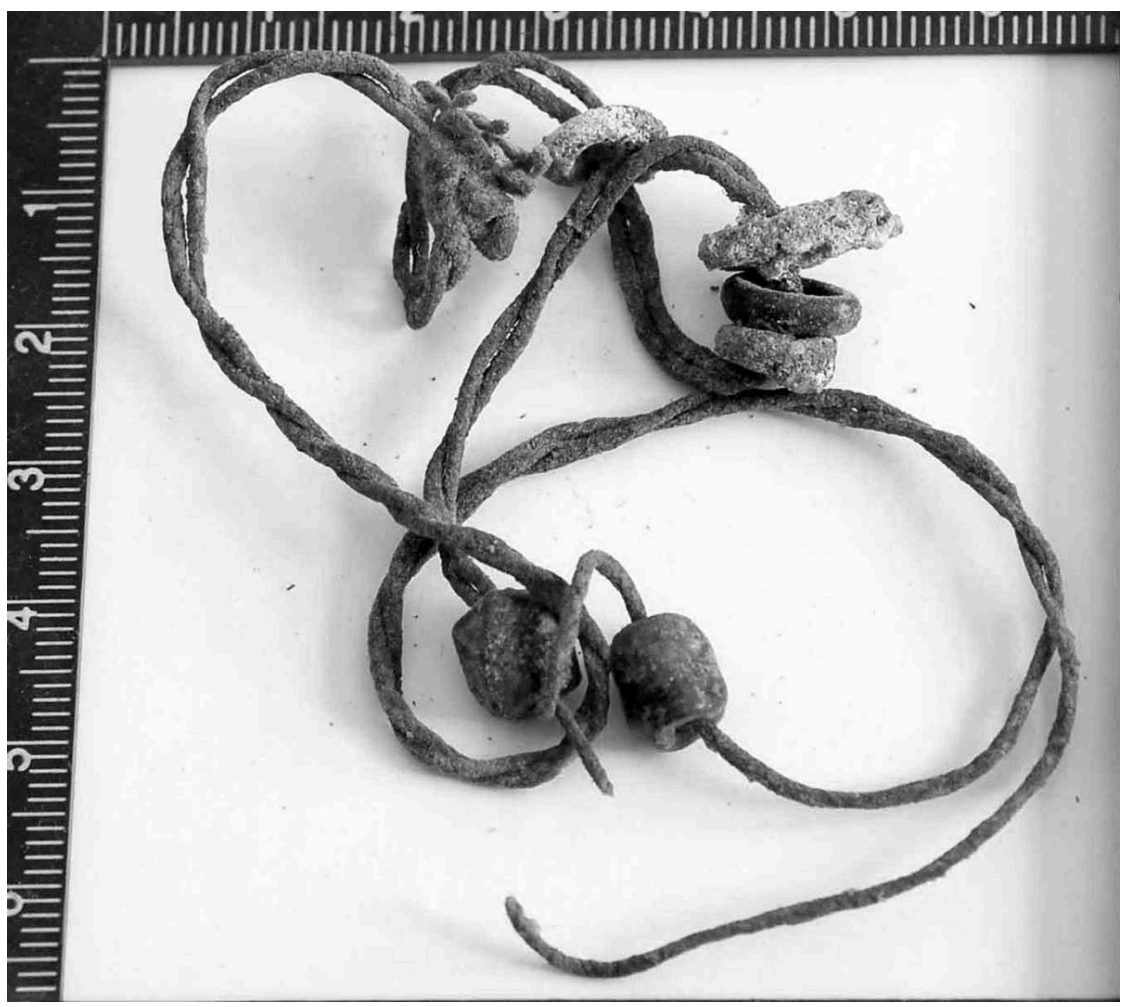

Photo F. Letellier-Willemin / Mission archéologique d'el-Deir 


\section{Production et usage du coton dans les textiles d'el- Deir : première esquisse}

19 Nous l'avons vu lors de l'inventaire, le coton est présent à el-Deir sous des formes variées évoquant différents usages. Il apparaît en très faible quantité dans les nécropoles dites "traditionnelles» et en grande quantité dans la nécropole dite "chrétienne ${ }^{4}$. Curieusement il n'a pas été mis en évidence dans les textiles des momies de chiens provenant de la tombe P5 du Piton aux Chiens, ni de la tombe E9 de la nécropole Est. Il n'a pas non plus été trouvé dans les sondages de la forteresse voisine.

Les fils montrent toujours une torsion en -S et les armures de tissage présentent une qualité très variable. La maîtrise de cette nouvelle fibre ne fut apparemment pas évidente. Cela pose de nouvelles questions quant à la nature des outils employés et l'expérience des fileurs et des tisserands, artisans professionnels ou bien particuliers. Sur le site, certains fragments de textiles en coton possèdent des départs de tissage et des décors de trame, caractéristiques de l'emploi d'un métier à poids. À bien des égards, ces tissus de coton rappellent les productions contemporaines en laine mises en évidence sur le site. Les Grecs, puis les Romains à leur suite, ont grandement contribué à développer la production et l'usage de la laine en Égypte à partir de l'époque ptolémaïque, en commençant par la région du Delta (Préaux 1939 : 95-98 et 106-112, Wipszyscka 1965 : 26-37 et chapitre IV). Il n'est donc pas très surprenant d'observer une certaine communauté technique entre les tissus fabriqués en laine et ceux en coton, toutes deux fibres courtes au comportement similaire. Au contraire, la différence est grande avec les textiles en lin, fruits de traditions et de savoir-faire millénaires. L'arrivée du coton à Kharga laisse supposer d'autres gestes, et d'autres types d'apprentissage.

Les conditions locales de production de ces tissus en coton ne nous sont pas encore connues. Toutefois, la présence de graines et de fils de coton dans l'environnement du temple nous permet d'évoquer l'hypothèse d'un atelier de tissage situé à proximité.

En tant que fibre végétale, les tissus de coton ne tombent pas sous l'interdiction qui frappe les étoffes de laine, et peuvent être admis dans les tombes. Issus de tissus ayant servi à la vie quotidienne, ils sont réemployés en domaine funéraire comme rembourrage autour des corps et dans les momies, ainsi qu'en support de cartonnages dans les nécropoles dites «traditionnelles ». Dans la nécropole dite "chrétienne », les rares linceuls en coton mis au jour ne sont pas neufs et ne nous sont parvenus que sous forme très fragmentaire. Soulignons à nouveau la quantité impressionnante de cordelettes funéraires en coton, dont l'origine et la fabrication restent à expliquer.

\section{Utilisation des fibres textiles à el-Deir : approche comparative}

Le lin, fibre identitaire dès les premières dynasties, symbolise une part importante des pratiques funéraires pharaoniques. On le retrouve donc sans grande surprise dans les nécropoles dites "traditionnelles» du site, où il représente la grande majorité des occurrences textiles. D'un point de vue technique, une grande partie de ces textiles en 
lin reflète une qualité constante, qu'il s'agisse des textiles du quotidien en réemplois, ou bien des linceuls neufs tissés à l'occasion des funérailles. Cette grande homogénéité s'observe sur une très longue durée, pendant huit cents ans pour le site d'el-Deir. On constate un changement de qualité notable dans le tissage des linceuls neufs de la nécropole dite "chrétienne ", à partir du III siècle $\mathrm{EC}$ : particulièrement visible au niveau des fils, cette moins bonne qualité se manifeste par des torsions et des diamètres irréguliers. Dans le même temps, la quantité de textiles en lin diminue pour faire place à ceux en laine et, dans une plus faible proportion, à ceux en coton. La laine s'impose alors peu à peu comme fibre de prédilection pour la confection des vêtements, parfois très luxueux, qui finissent plus tard leur vie comme dernière enveloppe protectrice du corps en milieu funéraire.

\section{Le coton dans la Grande Oasis} grandes qualités, qui ont de tout temps assuré sa popularité. Sa couleur blanche naturelle, son emploi sans préparation préalable, son aspect laineux, sa douceur et sa nature végétale semblent des critères positifs évidents, esthétiques, religieux et de confort. Nous pouvons supposer qu'il s'agit dans un premier temps d'un produit commercial, reconnu comme un matériau de valeur par les acteurs influents du textile. 
Sous quelle forme est-il proposé aux artisans et consommateurs d'el-Deir : sous forme de fibre, de fil, ou de textile? Quelle peut être la provenance de ce coton? L'existence d'une route terrestre reliant la Nubie à Kharga et, plus loin aux provinces égyptiennes des bords du Nil, constitue un argument de poids pour son origine africaine. Mais d'autres échanges pouvaient également exister avec l'Est, comme le confirme la présence dans le temple d'el-Deir d'un murex identique aux spécimens originaires de la Mer Rouge mis en évidence dans des forts romains du désert oriental (Cardon, comm. pers.). Finissons par évoquer enfin l'hypothèse d'une route vers l'ouest et le pays des Garamantes, où le coton est présent dans des tombes et des habitats de la même période (Mattingly et al. 2007 : 154, Pelling 2013).

Dans ce schéma de production, la culture locale du coton n'interviendrait alors que dans une deuxième phase. Le coton est un marqueur d'importants changements sur le site d'el Deir, dont l'étude n'est qu'à ses débuts.

\section{BIBLIOGRAPHIE}

Bagnall R. 2008 - Cotton and Economy of the Small Oasis. Bulletin of the American Society of Papyrologists 45 : 21-30.

Barakat H. \& Baum N. 1992 - La végétation antique de Douch (oasis de Kharga), une approche macrobotanique. Le Caire, Institut français d'archéologie orientale. (Document de fouille de l'Institut français d'archéologie orientale ; 27).

Dunand F. 2010 - El-Deir. Nécropoles I, La nécropole Sud. Paris, Cybèle éditions.

Dunand F. 2012 - El-Deir. Nécropoles II, Les nécropoles Nord et Nord-Est. Paris, Cybèle éditions.

Dunand F. 2015 - El-Deir. Nécropoles III, La nécropole Est et le piton aux chiens. Paris, Cybèle éditions.

Heurtel C. 2003-2004 - Tissage et tissus funéraires. Grafma Bulletin 7/8 : 60-62.

Jones J. \& Oldfield R. 2006 - A kind of wool is made by the Egyptians from a tree.... Archaeological Textiles Newsletter $43: 27-32$.

Kajitani N. 2006 - Textiles and their context in the third -to fourth- century CE, cemetery of alBagawat, Khargah oasis, Egypt, from the 1907-1931 excavations by the Metropolitan Museum of Art, New-York. In : Schrenk S. (Ed.) Textiles in situ: Their find spots in Egypt and neighbouring countries in the First Millenium CE. Abegg-Stiftung, Riggisberger Berichte 13 : 57-68.

Letellier-Willemin F. \& Médard F. 2012 - Techniques inattendues dans un fragment textile en coton du site d'El Deir, oasis de Kharga, désert occidental égyptien. Archaeological Textiles Newsletter 54 : 62-71.

Letellier-Willemin F. \& Moulherat C. 2006 - La découverte de coton dans une nécropole du site d'El Deir, oasis de Kharga, désert occidental égyptien. Archaeological Textiles Newsletter 43 : 20-27.

Livingstone R.J. 2009 - Late antique household textiles from the village of Kellis in the Dakhleh oasis. In : De Moor A. \& Fluck C. (Ed.) Clothing the house: furnishing textiles of the $1^{\text {st }}$ millennium from Egypt and neighbouring countries. Lannoo, Tielt : 73-85. 
Mattingly D., Lahr M. \& White K. 2007 - Desert Migrations: People, environment and culture in the Libyan Sahara. Lybian Studies $38: 34-35$ et 40.

Pelling R. 2013 - The archaeobotanical remains (Chapter 18) and Botanical data appendices (Chapter 28). In : Mattingly D.J., Daniels C.M., Dore J.N., Edwards D., Leone A. \& Thomas D.C. (Ed.), The archaeology of Fazzan, vol. 4, Survey and Excavations at Old Jarma (Ancient Garama) carried out by C.M. Daniels (1961-1969) and the Fazzan Project (1997-2001). Londres, Society of Lybian Studies : 473-494, 841-852.

Préaux C. 1939 - L'économie royale des Lagides. Bruxelles, Fondation Égyptologique Reine Elisabeth.

Tallet G. 2015 - Les cartonnages. In : Dunand F. (Ed.), El-Deir Nécropoles III. Paris, Cybèle éditions : 371-452.

Tallet G., Gradel C. \& Letellier-Willemin F. 2012 - "Une laine bien plus belle et douce que celle des moutons" à El-Deir (oasis de Kharga, Égypte) : le coton au cœur de l'économie oasienne à l'époque romaine. In : Guédon S. (Ed.) Entre Afrique et Égypte : relations et échanges entre les espaces du sud de la Méditerranée à l'époque romaine. Bordeaux, Ausonius : 119-141.

Wild J.-P. 2006 - Berenike: Archaeological textiles in context. In : Schrenk S. (Ed.) Textiles in situ: Their find spots in Egypt and neighbouring countries in the First Millenium CE. Abegg-Stiftung, Riggisberger Berichte $13: 175-184$.

Wild J.-P. 2008 - Cotton: the new wool. Qasr Ibrim Study Season 2008. Archaeological Textiles Newsletter 44 : 16-18.

Wild J.-P. 2013 - The first Indian carpets - a view from Berenike. In : De Moor A., Fluck C. \& Linscheid P. (Ed.) Drawing the threads together, Textiles and Footwear of the 1st millennium AD from Egypt. Lannoo, Tielt : 74-85.

Wipszycka E. 1965 - L'industrie textile dans l'Égypte Romaine. Warsaw.

Yvanez E. 2016 - Spinning in Meroitic Sudan: Textiles Production Implements from Abu Geili. Dotawo, A Journal of Nubian Studies 3 : 153-178.

\section{NOTES}

1. Pour une présentation générale du site d'el-Deir et de ces différentes localités, voir le chapitre d'introduction dans El-Deir Nécropoles I, publié par Françoise Dunand (Dunand 2010).

2. Toutes les photographies, prises par l'auteur, sont reproduites ici avec l'autorisation de la Mission archéologique d'el-Deir.

3. Il s'agit de deux fils de torsion S, tordus ensemble en $\mathrm{Z}$, pour former un fil plus épais et solide nommé retors.

4. L'étude complète de la place du coton au sein du corpus textile d'el-Deir n'en est qu'à ses débuts. Il ne nous est malheureusement pas encore possible de produire des statistiques probantes, fondées sur l'ensemble du matériel.

5. Non mentionnés dans la publication (Barakat \& Baum 1992) mais dans le rapport préliminaire de 1998 (non publié). 


\section{RÉSUMÉS}

La présence du coton à el-Deir (oasis de Kharga, désert occidental égyptien) est une découverte archéologique récente, essentiellement d'origine funéraire. Bien qu'attesté en très petite quantité, le coton et son étude permettent de mettre en évidence des catégories textiles aux qualités variées et aux usages divers. Il semble intéressant, par ailleurs, d'étudier son évolution qualitative sur le site, parallèlement aux deux autres fibres en présence, le lin et la laine. Cette nouvelle fibre apporte un éclairage nouveau sur la vie des habitants, leurs pratiques funéraires, leur économie, et questionne sur les changements possibles dans la gestion du territoire et du commerce durant l'Antiquité ( $v^{\mathrm{e}} \mathrm{s}$. AEC.- $\mathrm{v}^{\mathrm{e}} \mathrm{s}$. EC).

The presence of cotton in el-Deir (Kharga oasis, Egyptian Western desert) is a recent archaeological discovery, mainly of funerary origin. Despite its relative rarity, cotton and its study show the existence of different textile categories, distinguished by varied degrees of quality and diverse usages. It is also interesting to observe the evolution of cotton's quality on the site, comparing it to other - better attested - fibres, such as linen and wool. This new fibre sheds a new light of the daily life of the inhabitants, their funerary practices, their economy, and implies possible changes in agricultural management and trade patterns during Antiquity $\left(5^{\text {th }} \mathrm{c}\right.$. $\mathrm{BCE}-5^{\text {th }}$ C. CE).

\section{INDEX}

Mots-clés : coton, fibres, lin, laine, pratiques religieuses, économie, statut, identité culturelle Keywords : cotton, fibres, wool, linen, religious practices, economy, status, cultural identity

\section{AUTEUR}

\section{FLEUR LETELLIER-WILLEMIN}

Université de Limoges CRIHAM EA 4270

Mission archéologique d'el-Deir, oasis de Kharga, désert occidental égyptien - Projet MAHES, LabExArchimède

f.letellier.willemin@free.fr 